\title{
A new gustometer: Template for the construction of a portable and modular stimulator for taste and lingual touch
}

\author{
Camilla Arndal Andersen ${ }^{1,2} \cdot$ Lorenzo Alfine $^{3} \cdot$ Kathrin Ohla ${ }^{3,4} \cdot$ Richard Höchenberger $^{3,4}$
}

Published online: 3 December 2018

(C) Psychonomic Society, Inc. 2018

\begin{abstract}
Taste research has been hampered by technical difficulties, mostly because liquid taste stimuli are difficult to control in terms of timing and application area. Exact stimulus control requires a gustometer, but the existing devices are either not well-documented or rather inflexible. We designed a gustometer based on a computer-controlled, modular pump system, which can be extended via additional hardware modules - for example, for heating of the stimuli or sending and receiving triggers. All components are available for purchase "off the shelf." The pumps deliver liquids through plastic tubing and can be connected to commercially available or custom-made mouthpieces. We determined the temporal precision of the device. Onset delays showed minuscule variation within pumps $(S D<3 \mathrm{~ms})$ and small differences between pumps $(<4.5 \mathrm{~ms})$. The rise time was less than $2 \mathrm{~ms}(S D<2 \mathrm{~ms})$, and the dosage volume bias was only $2 \%$. To test whether hemitongues could be stimulated independently, we conducted a behavioral experiment. A total of 18 participants received tasteless stimuli to the left, right, or both sides of the tongue. The side of stimulation was correctly identified on $91 \%$ of trials, indicating that the setup is suitable for lateralized stimulation. Electroencephalographic responses to water and salty stimuli were recorded from two participants; the stimulation successfully evoked event-related responses, demonstrating the suitability of the device for use in electrophysiological investigations. We provide a Python-based open-source software package and a Web interface to easily operate the system. We thereby hope to facilitate access to state-of-the-art taste research methods and to increase reproducibility across laboratories.
\end{abstract}

Keywords Gustometer $\cdot$ Taste stimulator $\cdot$ Taste $\cdot$ Stimulator $\cdot$ Gustation $\cdot$ Flavor $\cdot$ Touch

Despite the growing interest in gustatory processing, owing to its significance in food preference and food intake, the sense of taste remains the least understood sensory system. A major contributing factor is the difficulty of presenting liquid taste (or flavor) stimuli in a controlled manner. However, precisely controlled stimulus delivery with steep onset flanks is necessary for behavioral response time measurements and electrophysiological investigations, in order to take full advantage of the high temporal resolution these methods can provide. Furthermore, exact

Richard Höchenberger

r.hoechenberger@fz-juelich.de

1 Division of Technology and Innovation, DuPont Nutrition \& Health, Brabrand, Denmark

2 Department of Engineering, Aarhus University, Aarhus, Denmark

3 German Institute of Human Nutrition Potsdam-Rehbruecke, Nuthetal, Germany

4 Institute of Neuroscience and Medicine (INM-3), Research Center Jülich, Jülich, Germany temporal alignment of stimuli across trials is vital to studies of evoked potentials, in which the summed responses of single trials are analyzed, and poor timing inevitably impairs signal-to-noise ratios. Precise stimulus control is also crucial to ensuring reproducible stimulation within and between participants and across experimental sessions. The solution is to automate stimulus delivery with the help of a programmable taste stimulator-a gustometer - instead of presenting the stimuli manually - for example, by using hand-operated pipettes.

Before gustometers became commercially available, studies on taste perception required the development and construction of custom-made devices. For example, the first gustatory eventrelated potentials to liquid stimuli were obtained almost 50 years ago using a hinged spoon pouring liquids onto the tongue (Funakoshi \& Kawamura, 1971). Since then, numerous devices for gustatory stimulation have been designed, be they for behavioral (Ashkenazi, Fritz, Buckley, \& Marks, 2004), hemodynamic (Veldhuizen, Bender, Constable, \& Small, 2007), or electrophysiological (Kobayakawa et al., 1996; see also Ohla, Busch, \& Lundström, 2012, for a review) investigations. 
Some researchers have presented stimuli via a sponge (Wada, 2005) or used syringe pumps to deliver liquids through a set of tubes directly onto the tongue (Andersen et al., 2018; Franken, Huijding, Nijs, \& van Strien, 2011). This approach concurrently activates the gustatory and lingual tactile systems. To isolate the gustatory response, other researchers have embedded taste stimuli within a stream of tasteless stimuli, thereby minimizing or even abolishing lingual somatosensory responses through adaptation and habituation. Kobal (1985), for example, used an olfactometer to deliver gaseous stimuli embedded in a constant flow of tasteless air to the mouth. Similarly, the gustometers used by Plattig, Dazert, and Maeyama (1988) and Gerull, Mrowinski, and Schilling (1984) embedded aqueous stimuli in a continuous flow of water. To ensure that consecutive stimuli did not mix or dilute, Kobayakawa et al. (1996) inserted small air bubbles before and after each stimulus, thereby separating taste stimuli from the tasteless background solution. However, replication of the previously mentioned setups is often impeded by only partial documentation and the lack of commercial availability of the devices used.

More recently, a gustometer that delivers liquids as clearly separated atomized spray pulses has become commercially available (GU002: Burghart, Wedel, Germany; see Iannilli, Beger, Fürer, \& Hummel, 2015, for a description of this device). With this system, liquid tastants are embedded in a regular series of water or rinse pulses, desensitizing tactile perception over time and thereby producing a purely unimodal taste stimulation, without concurrent tactile activation. The pulse sequence can be configured so as to exclude mixing of the stimuli and to provide sufficient temporal precision to elicit gustatory event-related potentials, which have been observed in different laboratories (see, e.g., Crouzet, Busch, \& Ohla, 2015; Iannilli, Broy, Kunz, \& Hummel, 2017; Tzieropoulos, Rytz, Hudry, \& le Coutre, 2013). The introduction of the Burghart gustometer, for the first time, offered users access to taste research, without requiring the resources or skills to build their own gustometer. However, its relatively poor dissemination - only a few research groups worldwide use the device-indicates that it comes with certain drawbacks. The acquisition and maintenance costs are not affordable to everybody. The number of pumps is fixed (a maximum of five pumps for tastants, two pumps for rinse). Further potential limitations are posed by the relatively inflexible proprietary control software and the requirement by the manufacturer that participants must maintain an upright position during stimulation (but cf., e.g., Iannilli, Singh, Schuster, Gerber, $\&$ Hummel, 2012, who used a modified version of the GU002 in an fMRI study).

Direct comparison of the results from different research groups is often difficult, since so many different stimulation approaches have been employed over the past decades. Moreover, the development and construction, but also the maintenance, of a taste stimulator often require substantial resources. We therefore developed a comparatively cheap gustometer that is sufficient for a variety of experimental applications. It delivers temporally precise and accurate stimuli with steep onset flanks as commonly required in behavioral and electrophysiological investigations. Furthermore, its portability allows for easy transport between different experimental locations, such as a behavioral lab and an fMRI scanning facility. The device is modular and can be assembled with commercially available parts. The number of syringe pumps is variable and can be adjusted to the number of stimuli required in the experiment. Syringes can be refilled automatically in the course of an experimental session. Furthermore, the functionality of the setup can be expanded with a multitude of additional modules - for example, for heating, digital input/output (I/O), and so forth. All components are easily replaceable, allowing for optimal hygienic practices. The gustometer can be equipped with custom-made mouthpieces. Stimuli can be delivered to participants in either upright or supine position. Lateralized stimulus delivery (i.e., to the left and right side of the tongue separately) can be achieved with a mouthpiece design presented in this article. We developed a software package (pyqmix) to control the gustometer. Its intuitive programmable Python interface allows for great experimental flexibility and excellent integration with existing neuroscientific software packages, such as PsychoPy (Peirce, 2009) or Expyriment (Krause \& Lindemann, 2014). Additionally, we developed a Web application (pyqmix-web) that simplifies common tasks such as filling and emptying the syringes without requiring any programming knowledge. The source code of both software packages is available free of charge, allowing users to modify and extend the functionality, if required.

With this publication we aim to provide a template for researchers desiring to assemble and operate a well-documented, versatile, modular, portable, computer-controlled gustometer for research purposes, which can be built at a low entry price and without the need for advanced technical skills.

\section{Method}

A full list of the required components to build the gustometer, along with a price list, is compiled in Table 1 . Note that the prices are based on quotes from December 2017, and that other vendors may offer the same or similar products. 


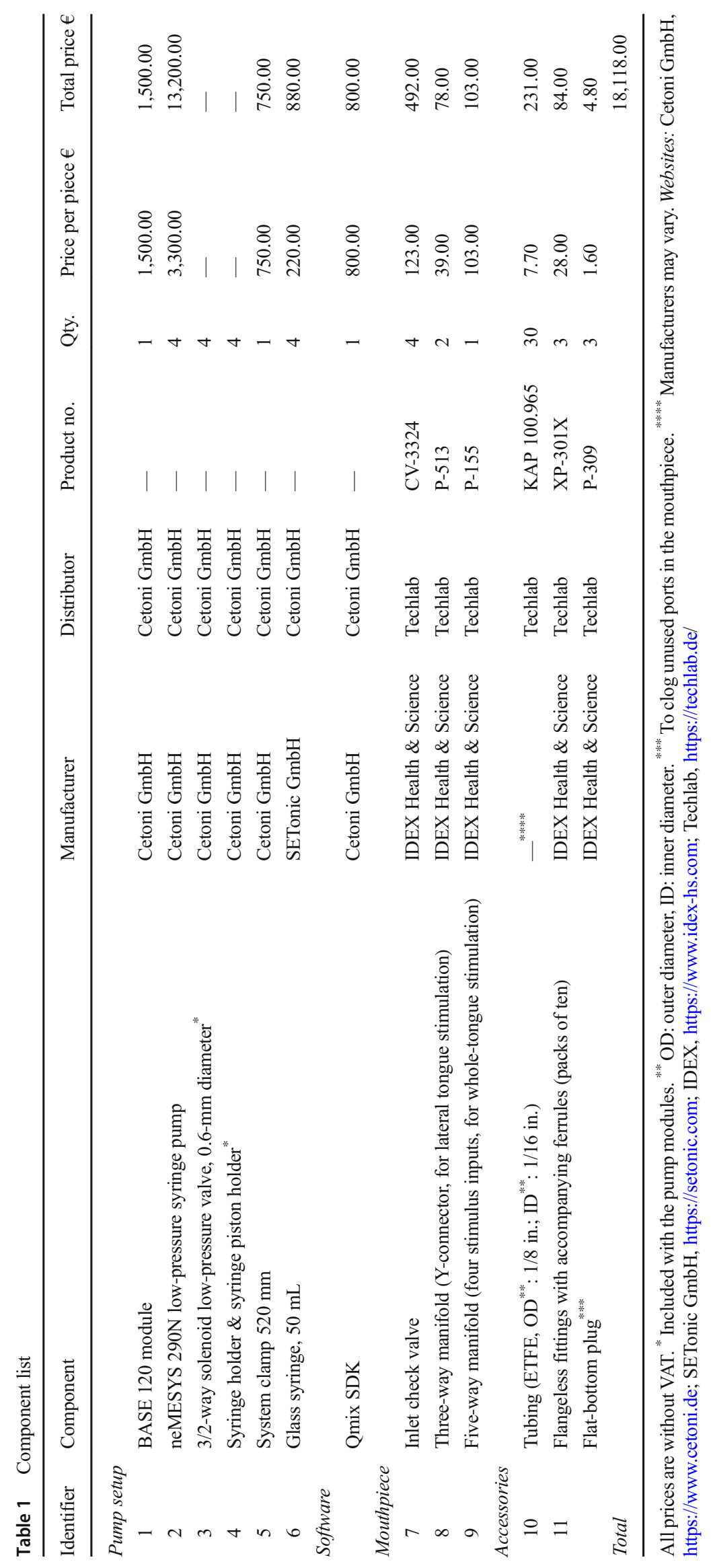




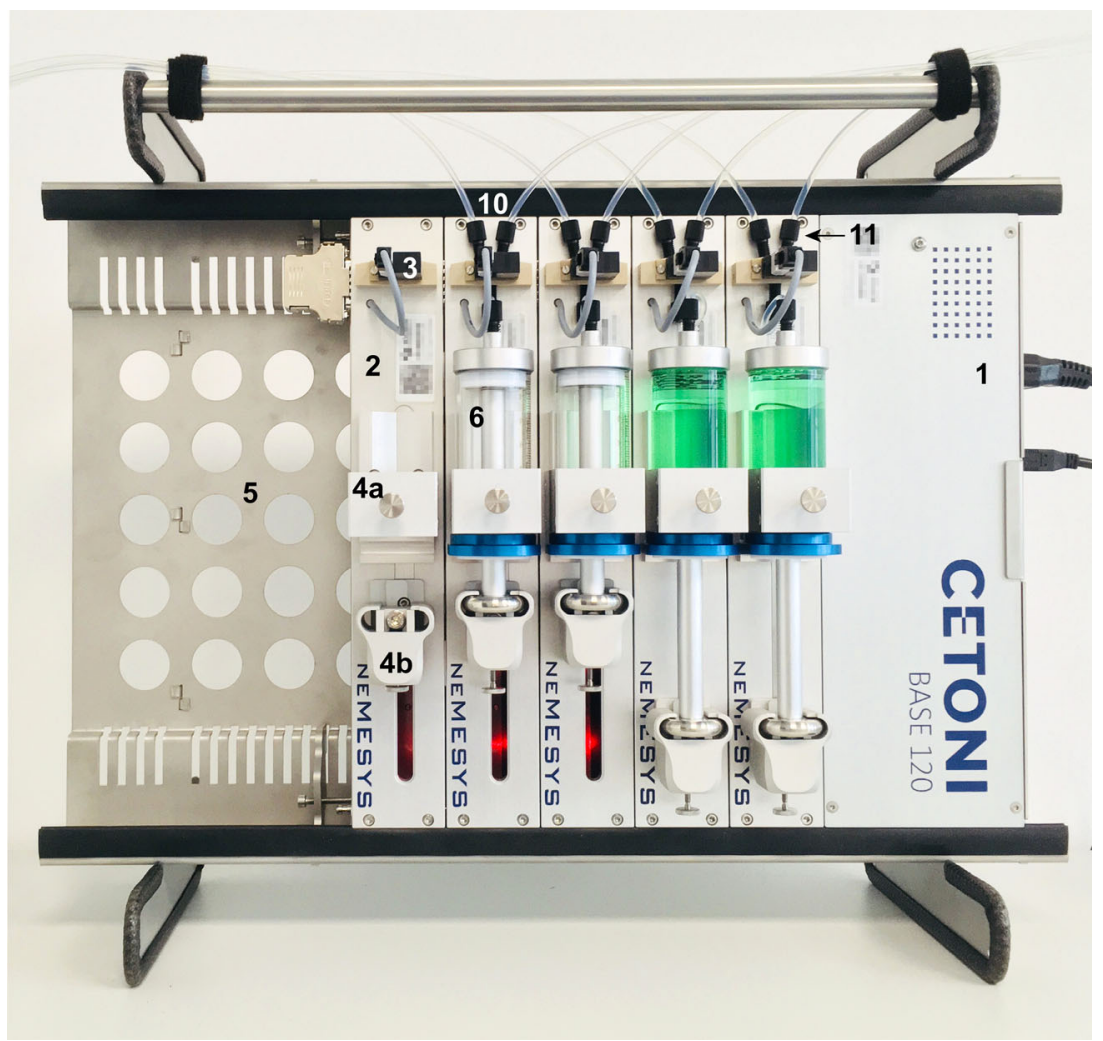

Fig. 1 Photograph of the gustometer. The BASE 120 module (1) and five neMESYS 290N low-pressure syringe pump modules (2) are mounted in a system clamp (5) in upright position. The installed 50-mL high-precision glass syringes (6) are held in place by syringe holders (4a) and syringe piston holders $(4 \mathrm{~b})$. The syringes themselves are connected to computer-controlled 3/2-way solenoid valves (3). Leak-proof tubing (10) connections are established through flangeless fittings with accompanying ferrules (11). The power cord and USB cable connect to the right side of the system. The component numbers used in this figure match the numbers in Table 1

\section{Gustometer construction}

The gustometer is modular, allowing the experimenter to adjust it to a number of experimental setups. In the following sections, we will present a configuration with two different mouthpieces, and we supplement the description with highlights of potential alterations and extensions of the system. Important design criteria were the portability of the device and straightforward assembly. Although the gustometer could be easily embedded in a cabinet, we implemented a version that can be easily carried and placed on a desk or a table with wheels, which improves accessibility and simplifies adaptations of the gustometer-for example, by the insertion or removal of a pump module.

\section{Pump system}

The heart of the gustometer is the computer-controlled, CE-certified neMESYS low-pressure syringe pump system (Cetoni GmbH, Korbussen, Germany). Its BASE 120 module powers up to eight neMESYS 290N lowpressure syringe pump modules and can be controlled by a PC via an integrated USB interface. The pumps are daisy-chained to the base module via serial ports with a termination plug at the terminal port (see Fig. 1).

As an alternative to the low-pressure pumps, which exert a maximum force of $290 \mathrm{~N}$, the manufacturer also offers much more powerful pump modules that deliver 1,000, 2,600, and even up to $7,000 \mathrm{~N}$ (neMESYS $1000 \mathrm{~N}$, neMESYS $2600 \mathrm{~N}$, and neMESYS $7000 \mathrm{~N}$, respectively), which may be used to deliver more viscous stimuli. The power supplied through the BASE module determines the type and number of pumps that can be operated simultaneously. For example, the BASE 120 module can drive up to eight low-pressure pumps, whereas the BASE 600 can operate 41.

The neMESYS syringe pumps can create uniform, pulsation-free fluid streams and accurately dose fluids down to the nanoliter range. The pumps can be used in sequential order (one after the other) and in parallel (multiple pumps acting simultaneously). Continuous flow can be generated by emptying and filling two or more syringes in alternating order (i.e., one set of pumps dispenses from filled syringes, while another set refills the previously emptied syringes). 
The functionality of the pump system can be widened further by a large assortment of supplementary Qmix modules, including input/output modules (which can be used for sending and receiving trigger signals), valve, heating, pressure monitoring, spectrometric, and camera modules (Cetoni GmbH, Korbussen, Germany).

\section{Syringes}

We installed $50-\mathrm{mL}$ high-precision glass syringes (SETonic GmbH, Ilmenau, Germany) in each pump to reduce the frequency at which syringe refill would be necessary during operation (Fig. 1). Smaller syringes (from $10 \mu \mathrm{L}$ to $50 \mathrm{~mL}$ ) can be installed as needed, offering an effective way to increase the pressure exerted by the pumps. ${ }^{1}$ This can be particularly useful when working with high-viscosity fluids. Alternatively, the manufacturer offers syringes made of stainless steel to handle even higher pressures; these syringes, however, do not allow for visual check of the fill level or identification of potentially trapped air bubbles. It should be noted that the universal syringe connectors on the neMESYS syringe pumps do not limit the user to one specific syringe manufacturer, but allow for selecting from among numerous types of syringes from different producers.

\section{System clamp}

The base and pump modules were mounted in a so-called system clamp, a metal bracket that positions the pumps and installed syringes in an upright position (Fig. 1). The vertical alignment is not required by the manufacturer, but it ensures that air bubbles will float up and accumulate at the top of the syringes, from where they can be easily removed by partially emptying the syringes.

\section{Computer-controlled valves}

Each syringe was connected to a computer-controlled 3/2way solenoid valve with three ports (Cetoni $\mathrm{GmbH}$, Korbussen, Germany; see Fig. 1). In this type of valve, one of the three ports is permanently open; this port is connected to the syringe. The other two ports act in a reciprocal manner: when one is opened, the other is automatically closed. The valve state is controlled by a computer, allowing the user to switch the opening of the ports as needed. A typical setup would be one port connected to the stimulus reservoir for stimulus refill and to use the

\footnotetext{
${ }^{1} \mathrm{p}=\mathrm{F} / \mathrm{A}$, where $\mathrm{p}$ is the pressure produced by the pump, $\mathrm{F}$ is a constant describing the force applied by the pump, and $\mathrm{A}$ is the surface area of the syringe plunger, which directly depends on the syringe diameter. With decreasing syringe diameter, the exerted pressure can increase while the applied force remains constant.
}

other port to deliver the stimulus to the participant by dispensing from the syringe. This setup additionally enables the experimenter to refill the syringes between consecutive stimulus deliveries. For convenience, we will henceforth name the port that is connected to the stimulus reservoir the inlet port, whereas the port that leads to the mouthpiece will be referred to as the outlet port.

\section{Tubing}

The inlet port of each valve was connected to a stimulus reservoir via 1-m ETFE ${ }^{2}$ tubing (1/16-in. inner diameter, 1/8-in. outer diameter; Techlab, Braunschweig, Germany), and each outlet port was connected to a custom-made mouthpiece via $3.2 \mathrm{~m}$ of tubing. Syringes were attached to the solenoid valves via $0.1 \mathrm{~m}$ of tubing.

\section{Tubing connections}

All tubing connections were established via flangeless fittings and their accompanying ferrules (IDEX Health \& Science LLC, Oak Harbor, WA/USA), to ensure tight and leak-proof links.

\section{Mouthpiece}

To deliver the stimuli onto the tongue, we used a mouthpiece with a spray head attached to the outlet of a manifold via a short stub of tubing. However, the mouthpiece itself does not require connection to any spray head, but rather allows the user to choose an outlet that is best suited to the experimental needs. For example, the stub of tubing can be attached to a custom-made anatomically shaped structure, or participants can even hold the stub of tubing gently between their teeth. There is virtually no limitation as long as the outlet can be connected with the tubing in a leak-proof manner. We opted for a spray head because it atomizes the liquid and evenly distributes it to a large surface area of the tongue.

More syringes, each supplied by a different stimulus reservoir, can be used in a multistimulus setup; here, the tubing from $n$ pumps would terminate in an $n+1$-way manifold with an inlet check valve (IDEX Health \& Science LLC, Rohnert Park, CA/USA) for each tube, to prevent backflow of the liquids, and an outlet of the user's choice-for example, a spray head (Burghart, Wedel, Germany). The system further allows for the researcher to attach multiple mouthpieces at once, which may be used to stimulate the left and the right sides of the tongue independently. We used this setup in combination with a

\footnotetext{
${ }^{2}$ Ethylene tetrafluoroethylene, a derivative of PTFE, commonly known by its brand name, Teflon.
} 


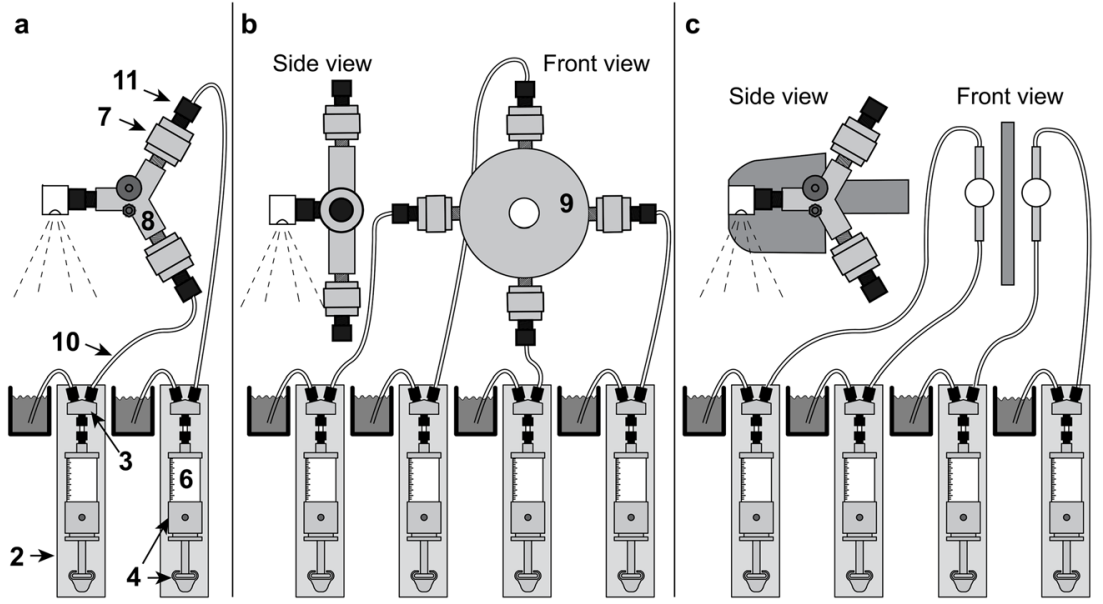

Fig. 2 Schematics of the gustometer setup: (2) pump module, (3) 3/2 solenoid valve, (4) syringe holder and piston holder, (6) glass syringe, (7) inlet check valve, (8) Y-connector, (9) five-way manifold, (10) tubing, and (11) fitting. (a) Setup for up to two stimuli. (b) Setup for up to four

separator that was placed along the midline of the tongue in a lateralized stimulation experiment (see the section of that name, as well as Figs. 2c and 3a).

\section{Control software}

The pump manufacturer, Cetoni, provides the proprietary Qmix Elements software that allows the user to control the pump system via a powerful graphical interface. This software allows for easy basic control of the system, and is even partly automatable. Yet, full integration into existing laboratory processes and experimental procedures often requires a much higher degree of flexibility. For example, the researcher may wish to align pump sequences with the presentation of visual stimuli or to synchronize pumping with laboratory hardware (e.g., electroencephalographic [EEG] amplifiers, magnetic resonance scanners) via transistor-transistor logic (TTL) pulses.

Fortunately, Cetoni also offers a Qmix software development kit (Qmix SDK). It exposes the full functionality of the pump system via a well-documented application programming interface (API) in the $\mathrm{C}++$ programming language. However, this language is not widely used in neuroscience and psychology labs - mostly because it is difficult to master without a background in computer science. We therefore decided to create a comprehensive and easy-to-use Python package, called pyqmix, that maps Python function calls to their corresponding C++ counterparts in the SDK. Over the past years, the Python programming language has progressively managed to establish itself as a free competitor for proprietary software like Matlab used for neuroscientific experimental control, thanks to powerful packages like PsychoPy (Peirce, 2009) and Expyriment (Krause \& Lindemann, 2014). As a full-scale programming language, Python offers a plethora of possibilities for presenting and manipulating stimuli, record and process participants' responses, and to control a large stimuli. (c) Setup for lateralized stimulation with up to two stimuli on each side of the tongue. A photograph of the mouthpiece is also shown in Fig. 3a

variety of laboratory hardware. The pyqmix software is free and open-source, released under the GNU General Public License (GPL). This means that the source code is available free of charge and can be modified by the user. The following code snippet

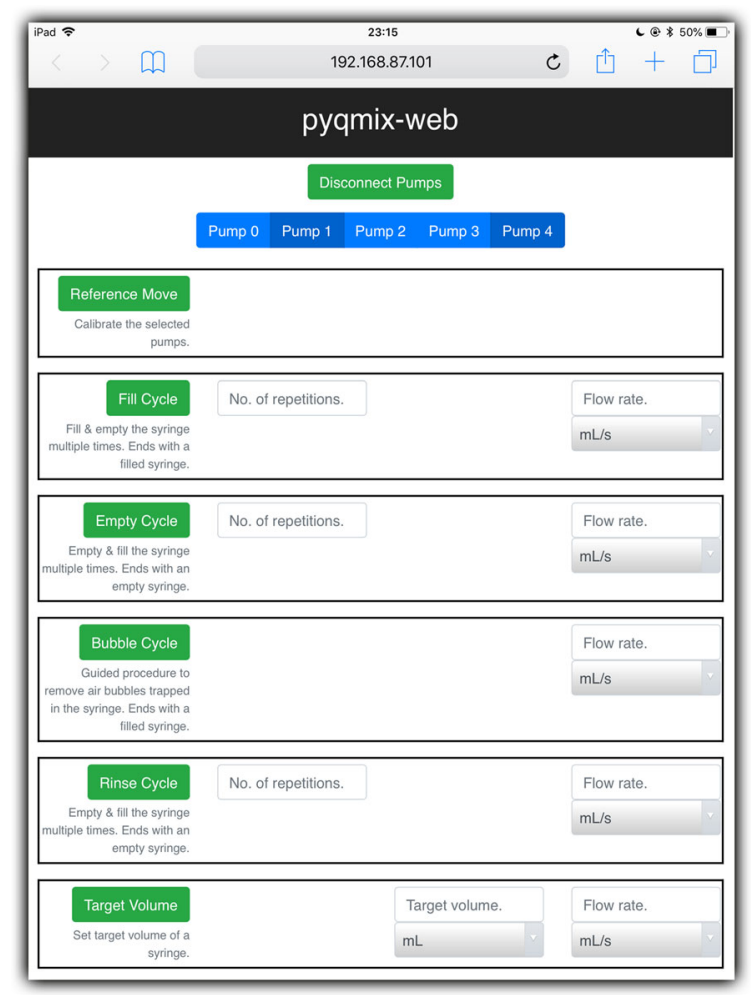

Fig. 3 Screenshot of the pyqmix-web interface running on an iPad. Five pumps have been detected in the gustometer. The user has selected Pumps 1 and 4 and may now calibrate the pumps (by executing a reference move), set the target volume, or initiate such pump activities as filling, emptying, rinsing, or the bubble cycle, to remove excess air from the syringes 
demonstrates how to fill a pump at a flow rate of $0.5 \mathrm{~mL} / \mathrm{s}$, followed by dispensing $1 \mathrm{~mL}$ at a flow rate of $1 \mathrm{~mL} / \mathrm{s}$.

pump.fill (flow_rate $=0.5$, wait_until_done=True) pump.dispense ('volume $=1$, flow_rate $=1$ )

For more complex examples, please refer to Appendices 1 and 2. Full documentation, including installation instructions, is available at http://pyqmix.readthedocs.org.

\section{Web interface}

To further simplify operation of the pumps, we implemented a remote-control interface that can be accessed via a Web browser (Fig. 4). This software, pyqmix-web, interfaces with pyqmix on either a local or a remote computer and allows it to perform common tasks in a straightforward way, including pump calibration (reference move) and filling, emptying, or rinsing of the syringes. Additionally, it implements a method for the removal of bubbles, which often get trapped in the syringes during initial filling. The Web application guides the user through all required steps of these procedures. No prior programming knowledge is required in order to use the app. We provide a self-contained executable at https://github.com/psyfood/pyqmix-web. No installation is required, since the executable contains all required dependencies, including Python and pyqmix. Like pyqmix, the software is free and open-source (GPL-licensed).

\section{Temporal properties}

To verify the suitability of the gustometer for experiments that demand precisely timed liquid stimulation with steep stimulus onset flanks, we measured two key temporal properties of stimulus delivery (onset delay and rise time) through a spray head.

An electrical circuit was used to detect stimulus onset delay and rise time (Fig. 5). For this, two electrodes, spaced $5 \mathrm{~mm}$ apart, were placed on a surface $5 \mathrm{~mm}$ below the spray head of the mouthpiece, illustrated in Fig. 2a. The surface was tilted $45^{\circ}$, allowing the liquid to drip off (see Kelling \& Halpern, 1986, for a similar procedure). A

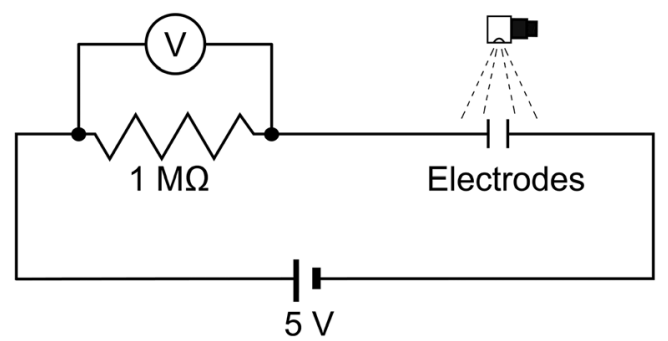

Fig. 4 Electrical circuit used for the measurement of onset delay and rise time of stimulus delivery
National Instruments USB 6212 data acquisition board (National Instruments, Austin, Texas, USA) provided a $+5-\mathrm{V}$ direct current voltage source to the circuitry and was used to register changes in electrical conductivity between the electrodes as the space between them was covered with liquid. The electrical conductivity between the electrodes was measured indirectly as the potential difference (in volts) across a $1-\mathrm{M} \Omega$ resistor at a sampling rate of $2000 \mathrm{~Hz}$.

Two $50-\mathrm{mL}$ syringes were attached to the pumps and filled with low concentration of salt water $(8.556-\mathrm{mM}$ sodium chloride - that is, $0.5 \mathrm{~g} / \mathrm{L}$ dissolved in deionized [DI] water). The two pumps were set to dispense $1 \mathrm{~mL}$ at $1 \mathrm{~mL} / \mathrm{s}$ in alternating order. The intertrial interval was $10 \mathrm{~s}$, to allow the surface between the electrodes to dry. The measurement of electrical conductivity between the electrodes was initiated as the pump was triggered to dispense and ended after $2.5 \mathrm{~s}$. Following each dispensing, the valve was switched from outlet to inlet position, to ensure sharp offsets. The procedure was repeated until both syringes were almost emptied, resulting in a total of 49 trials per pump.

The first four trials served to wet the electrodes. We determined the rise time and onset delay for each of the remaining 45 trials. Recordings were baseline-corrected by subtracting the mean voltage from 10 to $40 \mathrm{~ms}$ after pump initiation. The onset delay was defined as the time from pump initiation until $50 \%$ of the maximum voltage in that trial was reached. The rise time was defined as the time between $25 \%$ and $75 \%$ of the maximum voltage.

\section{Dosage precision and accuracy}

To verify that the gustometer delivered the desired volume, we collected ten samples of putative 1-mL dosages of distilled water at ambient temperature from one pump and determined the mass using a high-precision laboratory weighing scale (precision $=1 \mathrm{mg}$; Kern 572-30, Kern \& Sohn GmbH, Balingen, Germany). The collected volume was determined on the basis of the density of water at $20^{\circ} \mathrm{C}$, which is 0.998 $\mathrm{g} / \mathrm{mL}$.

\section{Lateralized stimulation experiment}

To test whether the hemitongues could be independently stimulated, which would facilitate studies of the lateralization of the gustatory processing pathway, and to show that the proposed stimulation elicited an evoked electrophysiological response, we conducted an experiment using two parallel mouthpieces (Figs. 2c and 3). 

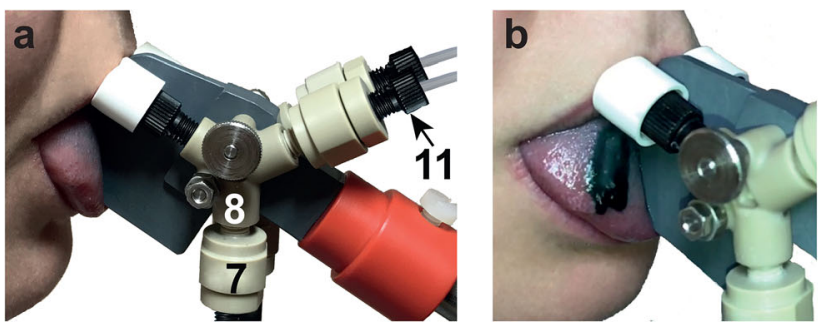

Fig. 5 (a) The mouthpiece used in the experiment. Lateralized stimulation is achieved through two separate spray heads placed on either side of a plastic separator (see also Fig. 2c for the schematics of the setup). (b) Demonstration of the lateralized stimulation locus using a colored liquid. Representative still images are extracted from a high-

Participants A total of 18 participants (age mean \pm standard deviation: $28.8 \pm 4.4$ years; 13 women, five men; one left-handed) took part in the study. Two participants (both 26 years old, female, right-handed) performing the experiment while EEG was recorded and are included as proof of concept. All participants were instructed to refrain from eating, drinking (except water), smoking, and brushing their teeth for at least $30 \mathrm{~min}$ prior to the experiment, to avoid any uncontrolled influences on orosensory perception (see Hummel, Genow, \& Landis, 2010; Iannilli et al., 2017; Jacquin-Piques et al., 2015, for similar procedures). The study was approved by the ethics board of the German Society for Psychology (DGPs) and conformed to the Declaration of Helsinki. All participants gave written and oral informed consent prior to the study and received monetary compensation.

Stimuli and apparatus A cushioned headrest was placed in front of a thin film transistor computer monitor at a distance of $60 \mathrm{~cm}$. A mouthpiece was attached to the headrest; it consisted of two three-way manifolds (two inlets, one outlet) placed on the left and right sides of a vertical, 5-mm-wide plastic separator (see Figs. 3a and 2c). The separator was to be positioned along the midline of the tongue and served to enable stimulation of the left and right sides of the tongue independently, without stimuli crossing sides. Each inlet port was connected to a dedicated syringe; deionized (DI) water was supplied to one port (serving as a rinse and as touch stimuli), and a salty solution (0.342-M sodium chloride; i.e., $20 \mathrm{~g} / \mathrm{L}$, dissolved in DI water) was supplied to the other port (serving as the taste stimuli; Fig. 2c). Spray heads were connected to the outlets of the manifolds. The pump system was placed outside of a sound-attenuated experimental booth.

Procedure Participants were seated in the sound-attenuated booth and were instructed to rest their forehead on the headrest and to protrude the tongue such that its anterior part was held against the separator, spatially separating the tongue at the midline, with the spray heads hovering approximately $5 \mathrm{~mm}$ above the tongue. The stimuli were sprayed onto the tongue and consecutively dripped off into a bowl, to avoid swallowing.

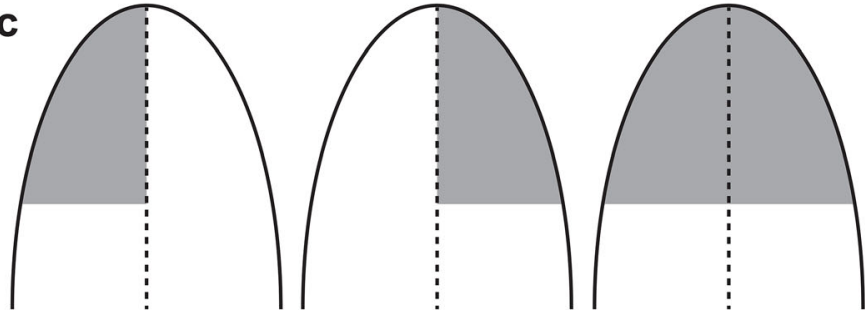

speed video recording (240 frames per second; iPhone SE, Apple Inc., Cupertino, CA/USA). (c) Illustration of the experimental conditions: stimulation of the left side, right side, and both sides of the tongue, in that order

Participants performed two interleaved tasks, a touch and a taste task. At the beginning of each trial, the type of the upcoming task was displayed on the computer monitor for $2 \mathrm{~s}$. Then a fixation cross appeared in the center of the screen. After a random duration of 1-2 s (selected from a uniform distribution), stimulus delivery was initiated for a duration of $1 \mathrm{~s}$ at a flow rate of $1 \mathrm{~mL} / \mathrm{s}$. In touch trials, water was delivered to the left, the right, or both hemitongues; in taste trials, salty solution was delivered to both hemitongues or only to the left or the right hemitongue, while water was sprayed onto the other; see Figs. $3 \mathrm{~b}$ and $3 \mathrm{c}$. This was done to ensure similar tactile stimulations across both sides of the tongue during taste trials. To ensure a sharp stimulus offset, the outlet ports of the valves were closed immediately after the pumps had stopped delivering the stimulus. Three seconds after stimulus offset, the computer monitor prompted participants to report the locus of stimulation (touch trials: side of tactile stimulation; taste trials: side of salty stimulation) by pressing one of three buttons on a keyboard (left arrow for left side, right arrow for right side, or down arrow for both sides). The response hand changed halfway through the experimental session, and the starting hand was counterbalanced across participants. A blank screen was presented immediately after registering a response, or after $5 \mathrm{~s}$ if no response was provided, and it stayed until the next trial started (for a minimum of $4 \mathrm{~s}$ in touch, and $7 \mathrm{~s}$ in taste, trials). The hemitongues were rinsed with $3 \mathrm{~mL}$ of water each during this intertrial interval (ITI) following taste trials. The interstimulus interval (ISI) between a touch and the consecutive taste stimulus was 11.0-13.0 $\mathrm{s}$, and the ISI between a taste and the consecutive touch stimulus was $14-16.9 \mathrm{~s}$. The water syringes were refilled during the ISI.

In total, 180 touch and 180 taste trials were presented in a pseudorandomized order, such that the same condition would not occur on more than two consecutive trials within each task. The experiment was split into six consecutive blocks with equal numbers of trials (i.e., 60 trials per block). The taste trials were recorded for use in another study and are only reported here for the two EEG participants.

To ascertain that stimulus delivery allowed for the recording of evoked potentials, we measured the electrophysiological 
response in the lateralized stimulation experiment using an actiCHamp amplifier system with $64 \mathrm{Ag} / \mathrm{AgCl}$ active channels positioned according to the extended 10-10 system (Brain Products GmbH, Munich, Germany). EEG data were sampled at $500 \mathrm{~Hz}$ and passed through an analog $0.01-\mathrm{Hz}$ high-pass and a 200-Hz low-pass filter using PyCorder (Brain Vision LLC, Morrisville, NC, USA). We sent a $+5-\mathrm{V}$ trigger pulse to the EEG amplifier via the National Instruments data acquisition board when the syringe pumps were started, marking the pump onset in the continuously recorded EEG stream.

Data analysis-Touch localization For each participant and condition, the proportions of left, right, and both responses were calculated. The results were then averaged across participants, yielding a grand mean confusion matrix. The statistical significance of the responses was evaluated by averaging the proportions of correct responses for each participant and comparing the results against chance level (33.3\%) using a one-sample $t$ test. Trials without a response were omitted from the analysis.

Data analysis-Evoked responses The EEG data were analyzed offline using MNE (version 0.16; Gramfort et al., 2013) in Python (version 3.6.6). First, we removed drifts in the data by linear detrending, and then applied a zero-phase high-pass and a low-pass hamming-windowed finite impulse response (FIR) filter, with cutoffs at 0.25 and $12 \mathrm{~Hz}$, respectively (transition width: $0.25 \mathrm{~Hz}$ for the high-pass filter and $3 \mathrm{~Hz}$ for the low-pass). The stimulus onset trigger times were shifted according to the stimulus onset delay, which was determined as described in the Temporal Properties section, and the data were segmented into epochs ranging from -0.2 to $1.0 \mathrm{~s}$ relative to stimulus onset.

On the basis of visual inspection, we interpolated excessively noisy channels and channels that showed large nonstereotypical artifacts in individual epochs. Epochs with unique, nonstereotypical artifacts were excluded $(<2 \%$ of epochs were removed). Stereotypical artifacts, such as ocular, cardiac, and muscle activity were removed by rejecting their corresponding independent components estimated from an independent component analysis (ICA) decomposition (FastICA; Hyvarinen, 1999) of data generated from an equivalent run of the preprocessing steps described above, except during the FIR filtering step, in which cutoff frequencies of 1 and $40 \mathrm{~Hz}$ were used, instead.

Finally, the data were re-referenced to the average of all channels; the mean of the 200-ms prestimulus baseline was subtracted from the signal; and the individual channels were interpolated in epochs that had been marked as problematic by Autoreject (Jas, Engemann, Bekhti, Raimondo, \& Gramfort, 2017). All touch and taste trials, separately, were averaged within participants. The reference-independent amount of electrical activity was calculated as the spatial standard deviation across channels at every time point (global field power; Lehmann \& Skrandies, 1980).

\section{Results and discussion}

\section{Temporal properties}

Onset delay Exact control of stimulus onset delay is paramount, to allow for temporally precise response time measures and take full advantage of the high temporal precision of electrophysiological recordings (EEG or magnetoencephalography). Stimuli were delivered with an onset delay of $44.1 \pm 2.6 \mathrm{~ms}$ (mean \pm standard deviation) from one pump and $48.3 \pm 2.9 \mathrm{~ms}$ from the other (Fig. 6). The near-constant onset delay suggests that the gustometer is indeed suitable for use in electrophysiological studies and obviates the need for complex online detection of stimulus onset.

Rise time Likewise, it is important that the gustometer deliver stimuli with a short rise time, to activate a large number of receptors in the targeted region simultaneously. The stimulus pulses delivered by our gustometer had a rise time of less than $2 \mathrm{~ms}$. Specifically, measurements showed rise times of $1.9 \pm$ $1.6 \mathrm{~ms}$ (mean \pm standard deviation) for one pump, and $1.9 \pm$ $1.2 \mathrm{~ms}$ for the other. The device is able to deliver stimuli with rise times as short as those from previously reported gustometers, albeit that the authors used differing definitions of rise time, or did not specify an exact definition at all $(<15$ ms, Crouzet et al., 2015; $<20 \mathrm{~ms}$, Kobal, 1985, and Kobayakawa et al., 1996; < 50 ms, Iannilli et al., 2015).

\section{Dosage precision and accuracy}

Dosage accuracy was high, since the dosed volumes only deviated slightly from the desired volume of $1 \mathrm{~mL}$. Specifically, we measured the average dispensed volume to be $0.98 \mathrm{~mL}$, suggesting a volume bias of approximately $2 \%$. Precision was excellent, with a standard deviation of just $0.01 \mathrm{~mL}$. Overall, the data show that the gustometer reliably delivered the desired volumes, demonstrating that the amount of delivered stimulus material can be precisely controlled.

\section{Lateralized stimulation experiment}

Touch localization Identification accuracy of the locus of stimulation was high $(91 \%)$ and significantly exceeded chance level $[t(17)=28.33, p<.001]$, corroborating that the liquids did not cross the separator (see Table 2 for a confusion matrix). On average, participants responded to $>98 \%$ of trials.

Evoked responses Two participants correctly reported the side of touch stimulation in $99 \%$ and $95 \%$ of trials, respectively (Participants 1 and 2). For taste stimulation, the proportions of correct localizations were $90 \%$ and 


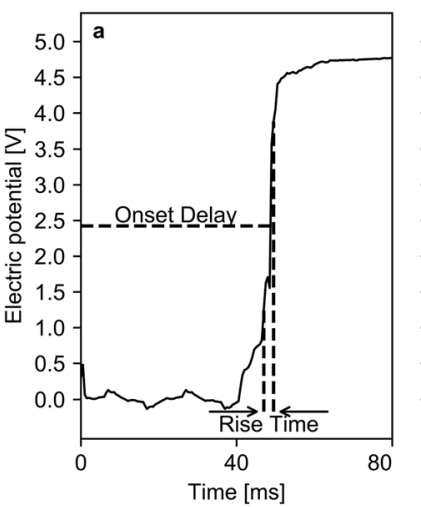

Fig. 6 Onset delay and rise time measurements. Pumping was initiated at time point zero and stopped after 1,000 ms, at which time the valves were switched to the inlet position. We measured the electric potential between two electrodes positioned below the spray head. (a) Sample measurement from one pump. The oscillations before approximately $40 \mathrm{~ms}$ are $50-\mathrm{Hz}$

$83 \%$. The mean global field power after touch and taste stimulation was derived for both participants; traces are shown in Fig. 7. The taste signal amplitudes exceeded the touch amplitudes for most time points. These data show that the device is indeed suitable for stimulation in EEG experiments, since it allows for successfully eliciting evoked potentials.

\section{Possible extensions and design alterations}

The modularity of the gustometer makes it possible to extend and alter its functionality in many ways.

Greater number of stimuli The gustometer can easily be extended to include more stimuli by adding pumps and adjusting the manifold in the mouthpiece.

Type of mouthpiece The gustometer does not impose the use of a mouthpiece of any specific type or shape; in fact, it can be used without any mouthpiece at all. The manner and site of stimulus delivery can therefore be fully adjusted to the experimenter's needs, be they to stimulate the tongue uni- or bilaterally, to spray, or simply to let the stimulus flow onto the tongue.

Table 2 Confusion matrix of behavioral responses from the touch localization task

\begin{tabular}{llll}
\hline \multicolumn{4}{l}{ Percentage of Responses } \\
\hline Stimulus Location & Left & Both & Right \\
Left & $96.0(4.5)$ & $3.4(4.2)$ & $0.7(1.2)$ \\
Both & $8.9(13.4)$ & $85.5(19.1)$ & $5.6(8.4)$ \\
Right & $2.3(4.5)$ & $5.3(4.9)$ & $92.4(7.3)$ \\
\hline
\end{tabular}

Values in parentheses are standard deviations

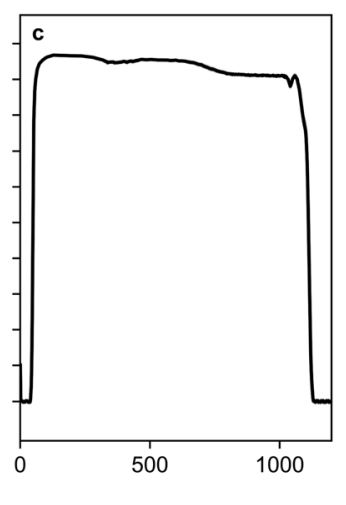

line noise artifacts in the measurement circuit. (b) Average signal onsets across all measurements for two pumps. (c) Average signal across all measurements for one pump. The shaded areas in panel $b$ depict the $95 \%$ confidence intervals, derived via bootstrapping (1,000 resamples)

Controlled stimulus temperature Cetoni offers heating and cooling modules. Warming the stimuli to tongue temperature can effectively avoid lingual temperature sensations. Alterations of stimulus temperature may also be used to study the effect of temperature differences on flavor perception.

Continuous stimulation In the experiments presented here, stimuli were delivered without preceding tactile stimulation, evoking both a gustatory and tactile sensation, much like in everyday eating situations. However, stimuli may also be embedded in an ongoing flow of "background rinse," avoiding the concurrent onsets of gustatory and tactile sensation.

Electric triggers The gustometer can be equipped with an input/output module (Qmix IO-B), which allows for generating and receiving electrical trigger signals in order to interface with other laboratory devices, including EEG and fMRI systems.

Viscous stimuli The gustometer can deliver viscous stimuli by employing the mid-pressure variant of the neMESYS syringe pump system (Cetoni GmbH, Korbussen, Germany) or alternatively, by using syringes with smaller diameter (since the generated pressure increases inversely proportionally with the cross-section area of the syringe).

\section{Advantages of our gustometer}

The main advantages of our gustometer are presented in the following section.

Precision and accuracy The gustometer precisely doses stimuli with a practically constant onset delay, steep 


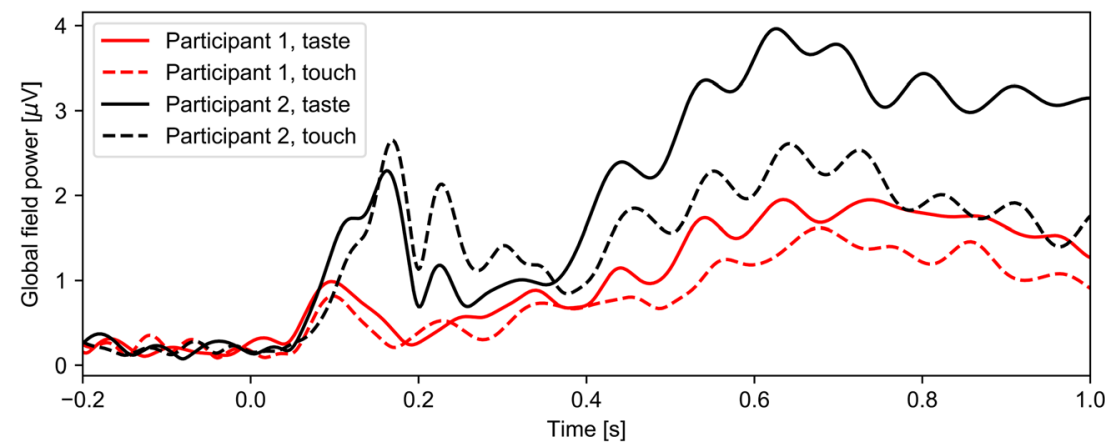

Fig. 7 Mean global field power for touch and taste stimulations. For both participants, the taste signal exceeded the touch signal over large periods of time

stimulus onset flanks, and accurate volume. These properties represent essential requirements of most behavioral, psychophysiological, and neuroimaging experiments, and they ensure stimulation replicability across trials and participants.

Automation The gustometer is computer-controlled. Our free and open-source Python-based software package allows the user to control the syringes within any experiment programmed in Python-for example, using PsychoPy (Peirce, 2009) or Expyriment (Krause \& Lindemann, 2014). This includes refilling the syringes during a running experiment, which allows for the delivery of large stimulus volumes and/or large numbers of stimuli, and even for continuous stimulation when using two pumps interchangeably.

Modularity The gustometer offers fully user-customizable setups. The experimenter can connect a variable number of mouthpieces and modules, depending on the experimental requirements at hand (for further information, please refer to the previous section: Possible Extensions and Design Alterations).

Hygiene Since all components of the gustometer are commercially available "off the shelf" and accessible, they can be easily replaced, offering excellent hygiene and minimizing tastant contamination between experiments.

Portability The gustometer is portable, because of its comparably small dimensions and also because it does not require compressed air or other fixed installations. It can therefore easily be set up in different experimental locations, and if installed on a wheeled table or cart, it can even be made mobile.

Low initial costs The user can opt to purchase a smaller version of the gustometer with only a few pumps at first, and to expand the gustometer later as more funds become available or experimental complexity increases.

\section{Best practices}

During construction and testing of the gustometer, we discovered several potential pitfalls that can be easily averted. We therefore compiled this collection of best practices to ensure reliable, precise, and accurate operation.

Avoid air bubbles Upon initial filling, air from the inlet tubing ends up in the syringes and must be removed before the experiment commences; otherwise, the precision and accuracy of stimulus delivery are compromised by the compressibility of the air in the syringe. A viable method to remove air from the syringes is to fill and dispense liquid repeatedly while the syringe is in an upright position. This procedure, at the same time, removes air from the outlet tubing. Once the syringes and tubing are completely filled with liquid, the gustometer is ready for use in an experiment. There will be no further accumulation of air, even during refilling, as long as the inlet tubes stay submerged in liquid and the refill flow is sufficiently slow.

Dispense after filling We observed that (re)filling of the syringes altered the onset delay of the first few subsequent dispense operations. This bias can be effectively avoided by dispensing a small volume after each filling procedure-for example, as a part of a tongue rinse, a break, or during any other period of the experiment in which the onset delay is not critical.

Mount syringes tightly To ensure the temporal precision of the gustometer, the position of the syringe piston holder must be checked, and possibly adjusted, every time a syringe is attached to a pump. The holder should fit very tightly. Please also consider that there may be small manufacturing differences, even between syringes of the same size.

Select the appropriate pump modules If the force required to move the syringe piston exceeds the maximum force 
the can be produced by the pump module, the motor stops and the pump switches into a fault state, meaning it will remain nonoperational until reset. It is therefore important to select the pump modules according to the specific experimental requirements. Low-pressure pumps are ideal for delivering liquids with low viscosity (like water) at moderate flow rates through relatively short tubes. If the experimenter desires to use stimuli with higher viscosity (e.g., oil), or to deliver liquids at high flow rates and through long tubes, mid-pressure pumps are highly recommended.

Keep the tubing short Each centimeter of tubing adds additional friction to the liquid flow, potentially influencing the onset delay, rise time, and pump force required. Long inlet tubes limit the flow rate at which the syringe can reliably be filled without producing air bubbles. Therefore, always ensure that all tubes used are no longer than absolutely necessary.

Close valves after stimulation Temporally precise stimulus offset can be achieved by closing the valve outlet immediately after pumping has stopped. Potential excess pressure in the system will force the remainder of the stimulus back into the stimulus reservoir instead.

Rinse after use Pumps and tubing should be rinsed after each recording session. Because the pumps can be programmed to fill and dispense automatically, it is possible to automate this procedure and conduct a thorough rinse of the system - for example, with ethanol or distilled water.

Revise temporal properties Several factors may influence the rise time and onset delay of the gustometer, including tubing length, syringe size, viscosity of the liquids, flow rate, dispensed volume, distance between tongue and mouthpiece, and so forth. It is therefore important to note that a rise time and onset delay are setupspecific and must be reevaluated whenever modifying the central parts of the system.

Avoid online mixing When attaching multiple syringes to the same manifold or mouthpiece, it might be tempting to mix stimuli "online" by dispensing from two or more syringes simultaneously. Depending on the specific type of mouthpiece used, this procedure might lead to unpredictable effects, since there is no way to ensure that the stimuli are being mixed properly before reaching the tongue. We therefore suggest avoiding online mixing if possible, and to test the success of the mixing procedure very carefully in cases in which mixing is unavoidable.

\section{Conclusion}

We have presented a gustometer that meets the demands of behavioral, electrophysiological, and hemodynamic investigations. Our measurements showed that stimulus timing and dosage volume were precise and accurate. Stimuli were delivered with steep onset flanks. We further demonstrated how the device can be used in lateralized behavioral testing, delivering stimuli to the left and right hemitongues independently. Although other researchers have used syringe pump systems cheaper than the one presented here in electrophysiological studies (e.g., Andersen et al., 2018; Franken et al., 2011), the highprecision pumps of our gustometer provide several advantages. By delivering higher flow rates, they enable the experimenter to produce a fine spray without requiring an additional supply of compressed air. The fully disclosed design and freely available control software (including the publicly available source code) make adjustments and design alterations for different experimental requirements easy. This versatility is further enhanced through the ability to add additional hardware modules to the system - for example, for digital I/O and stimulus heating. We hope that the great flexibility of the gustometer, its simple construction and operation, and the relatively low entry price will encourage more scientists to join the community of taste research and enrich this widely unexplored field with new and exciting discoveries.

Author note The authors thank Thomas Roeder (German Institute of Human Nutrition Potsdam-Rehbruecke, DIfE) for help with the mouthpiece design and development of the tongue separator. Andrea Katschak (DIfE) helped with data collection. Preben Kidmose (Aarhus University, Denmark) assisted in development of the electrical circuit used for timing measurements. The gustometer was designed and the behavioral and EEG data were collected at DIfE. The timing measurements were conducted, all data were analyzed, and the manuscript was written after K.O. and R.H. had moved to the Research Center Jülich. The authors declare no conflicts of interest.

Data availabiliy All timing measurements, the scripts used to perform these measurements, all behavioral touchdata, the behavioral taste data from participants 1 and 2, and the example scripts from the Appendix are deposited online at Zenodo and can be accessed via https://doi.org/10.5281/ zenodo.1313034. The sources of pyqmix and pyqmix-web are available at https://github.com/psyfood/pyqmix and https://github.com/psyfood/pyqmix-web, respectively. 


\section{Appendix 1 Pyqmix usage example with one pump}

The following code snippet illustrates a basic use case of pyqmix to initialize a pump, fill it, and dispense the liquid in ten $1 \mathrm{~mL}$ aliquots at a flow rate of $1 \mathrm{~mL} / \mathrm{s}$ with an inter-stimulus interval of approximately $2 \mathrm{~s}$.

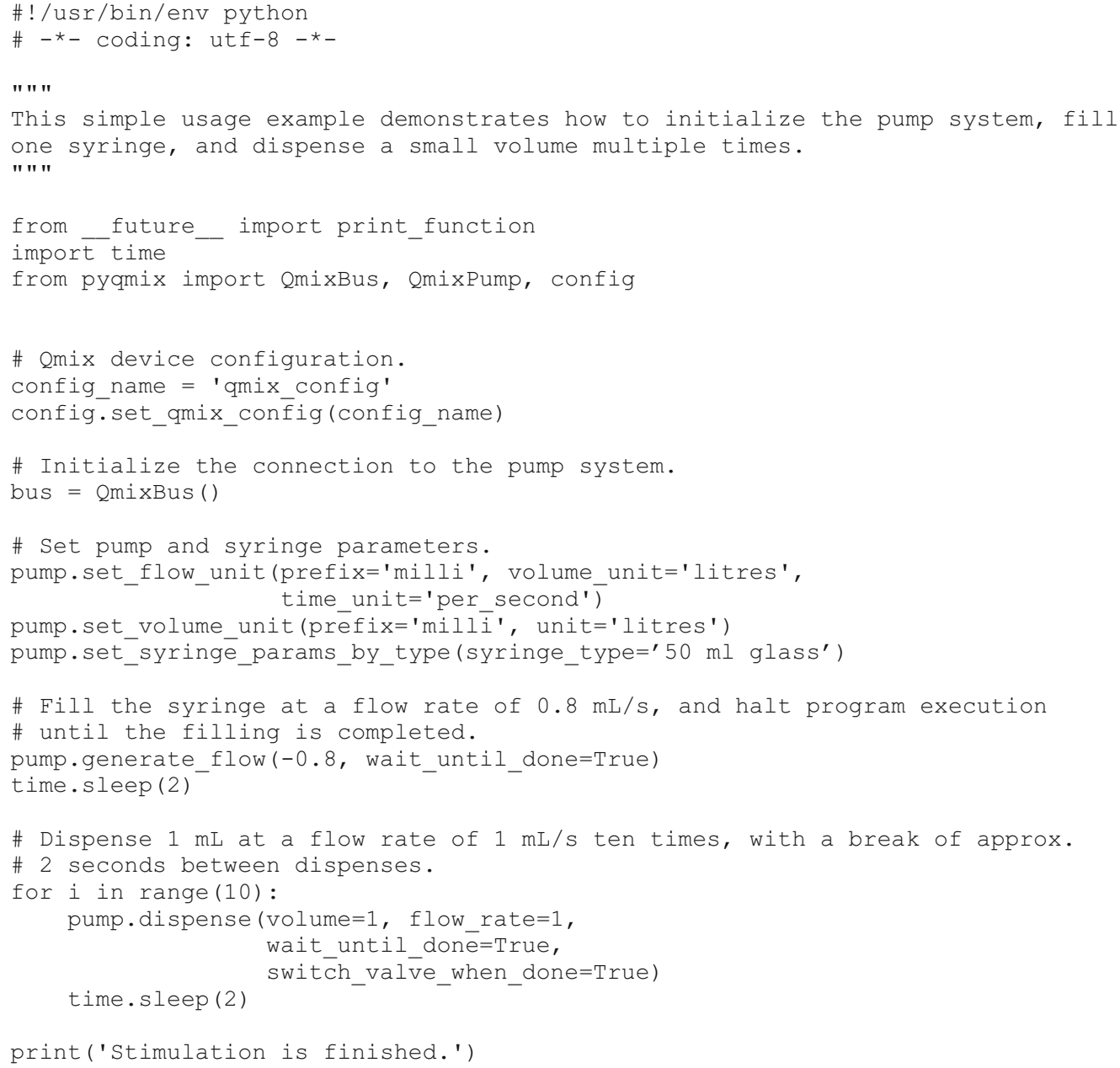




\section{Appendix 2 Pyqmix usage example with two pumps}

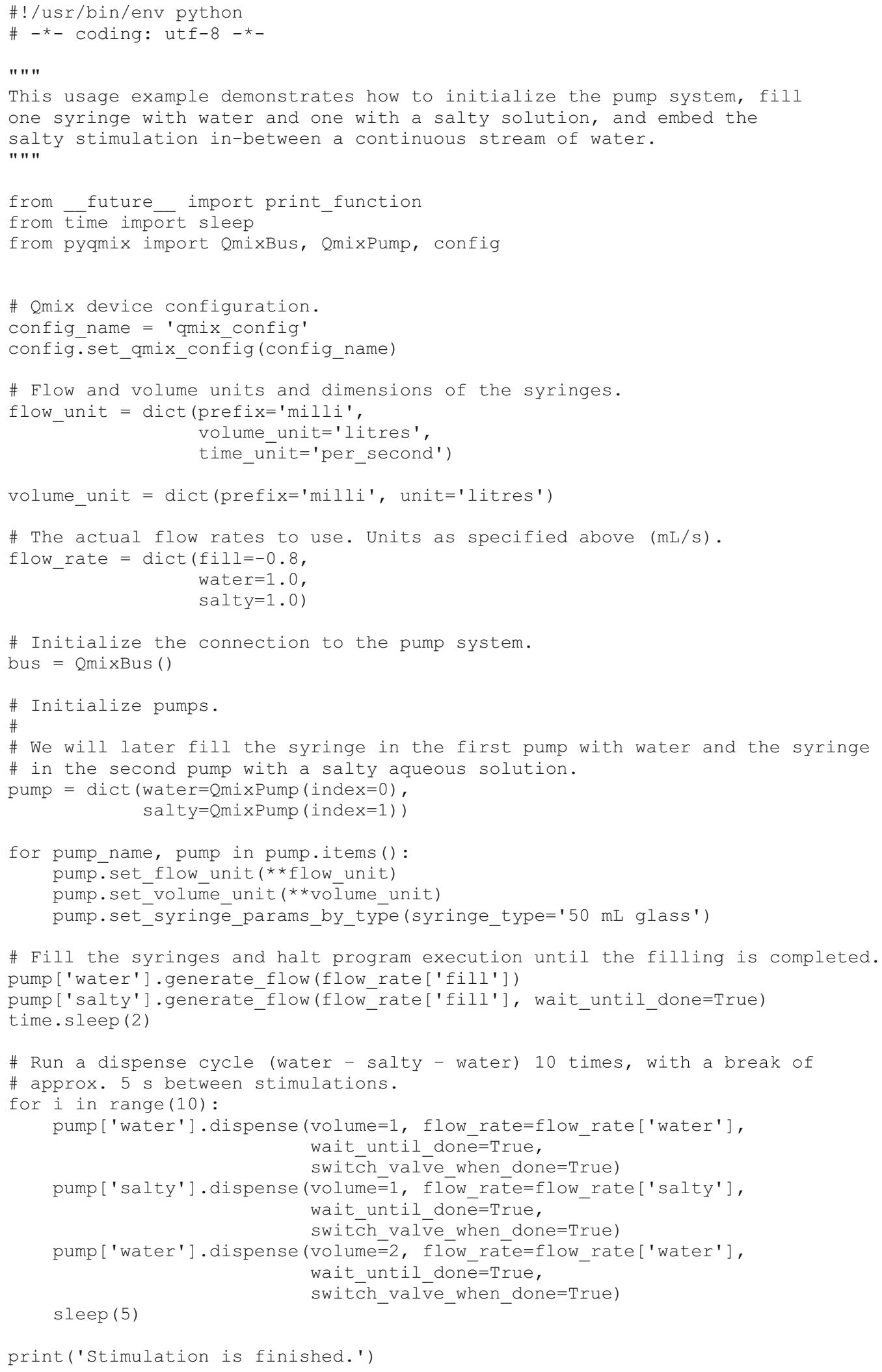




\section{References}

Andersen, C. A., Kring, M. L., Andersen, R. H., Larsen, O. N., Kjaer, T. W., Kidmose, U., . . Kidmose, P. (2018). EEG discrimination of perceptually similar tastes. Journal of Neuroscience Research. https://doi.org/10.1002/jnr.24281

Ashkenazi, A., Fritz, M., Buckley, J., \& Marks, L. E. (2004). The Temporal Automated System for Taste Experiments (TASTE). Behavior Research Methods, Instruments, \& Computers, 36, 8388. https://doi.org/10.3758/BF03195552

Crouzet, S. M., Busch, N. A., \& Ohla, K. (2015). Taste quality decoding parallels taste sensations. Current Biology, 25, 890-896.

Franken, I. H. A., Huijding, J., Nijs, I. M. T., \& van Strien, J. W. (2011). Electrophysiology of appetitive taste and appetitive taste conditioning in humans. Biological Psychology, 86, 273-278. https://doi.org/ 10.1016/j.biopsycho.2010.12.008

Funakoshi, M., \& Kawamura, Y. (1971). Summated cerebral evoked responses to taste stimuli in man. Electroencephalography and Clinical Neurophysiology, 30, 205-209.

Gerull, G., Mrowinski, D., \& Schilling, V. (1984). Objektive Gustometrie mit adäquaten Reizen durch Registrierung der Contingent Negative Variation [Objective gustometry with appropriate stimuli through registration of contingent negative variation]. EEG-EMG Zeitschrift für Elektroenzephalographie, Elektromyographie und Verwandte Gebiete, 15, 121-126.

Gramfort, A., Luessi, M., Larson, E., Engemann, D. A., Strohmeier, D., Brodbeck, C., ... Hamalainen, M. (2013). MEG and EEG data analysis with MNE-Python. Frontiers in Neuroscience, 7, 267. https:// doi.org/10.3389/fnins.2013.00267

Hummel, T., Genow, A., \& Landis, B. N. (2010). Clinical assessment of human gustatory function using event related potentials. Journal of Neurology, Neurosurgery and Psychiatry, 81, 459-464. https://doi. org/10.1136/jnnp.2009.183699

Hyvarinen, A. (1999). Fast and robust fixed-point algorithms for independent component analysis. IEEE Transactions on Neural Networks, 10, 626-634. https://doi.org/10.1109/72.761722

Iannilli, E., Beger, M., Fürer, R., \& Hummel, T. (2015). A gustatory stimulator. Journal of Neuroscience Methods, 255, 12-16.

Iannilli, E., Broy, F., Kunz, S., \& Hummel, T. (2017). Age-related changes of gustatory function depend on alteration of neuronal circuits. Journal of Neuroscience Research, 95, 1927-1936. https://doi.org/ 10.1002/jnr.24071

Iannilli, E., Singh, P. B., Schuster, B., Gerber, J., \& Hummel, T. (2012). Taste laterality studied by means of umami and salt stimuli: An fMRI study. NeuroImage, 60, 426-435. https://doi.org/10.1016/j. neuroimage.2011.12.088
Jacquin-Piques, A., Gaudillat, S., Mouillot, T., Gigot, V., Meillon, S., Leloup, C., . . . Brondel, L. (2015). Prandial states modify the reactivity of the gustatory cortex using gustatory evoked potentials in Humans. Frontiers in Neuroscience, 9, 490. https://doi.org/10.3389/ fnins.2015.00490

Jas, M., Engemann, D. A., Bekhti, Y., Raimondo, F., \& Gramfort, A. (2017). Autoreject: Automated artifact rejection for MEG and EEG data. NeuroImage, 159, 417-429. https://doi.org/10.1016/j. neuroimage.2017.06.030

Kelling, S. T., \& Halpern, B. P. (1986). The physical characteristics of open flow and closed flow taste delivery apparatus. Chemical Senses, 11, 89-104.

Kobal, G. (1985). Gustatory evoked potentials in man. Electroencephalography and Clinical Neurophysiology, 62, 449454.

Kobayakawa, T., Endo, H., Ayabe-Kanamura, S., Kumagai, T., Yamaguchi, Y., Kikuchi, Y., . . . Ogawa, H. (1996). The primary gustatory area in human cerebral cortex studied by magnetoencephalography. Neuroscience Letters, 212, 155-158

Krause, F., \& Lindemann, O. (2014). Expyriment: A Python library for cognitive and neuroscientific experiments. Behavior Research Methods, 46, 416-428. https://doi.org/10.3758/s13428-013-0390-6

Lehmann, D., \& Skrandies, W. (1980). Reference-free identification of components of checkerboard-evoked multichannel potential fields. Electroencephalography and Clinical Neurophysiology, 48, 609621.

Ohla, K., Busch, N. A., \& Lundström, J. N. (2012). Time for taste-A review of the early cerebral processing of gustatory perception. Chemosensory Perception, 5, 87-99.

Peirce, J. W. (2009). Generating stimuli for neuroscience using PsychoPy. Frontiers in Neuroinformatics, 2, 10. https://doi.org/10.3389/neuro. 11.010.2008

Plattig, K. H., Dazert, S., \& Maeyama, T. (1988). A new gustometer for computer evaluation of taste responses in men and animals. Acta Oto-Laryngologica, 458(Suppl), 123-128.

Tzieropoulos, H., Rytz, A., Hudry, J., \& le Coutre, J. (2013). Dietary fat induces sustained reward response in the human brain without primary taste cortex discrimination. Frontiers in Human Neuroscience, 7, 36. https://doi.org/10.3389/fnhum.2013.00036

Veldhuizen, M. G., Bender, G., Constable, R. T., \& Small, D. M. (2007). Trying to detect taste in a tasteless solution: Modulation of early gustatory cortex by attention to taste. Chemical Senses, 32, 569581.

Wada, M. (2005). Evoked response to taste stimulations. International Tinnitus Journal, 11, 43-47. 